\title{
PC Proxy: a Method for Dynamical Tracer Reconstruction
}

\author{
Peter Mills \\ peteymills@hotmail.com
}

September 4, 2019

\begin{abstract}
A detailed development of the principal component proxy method of dynamical tracer reconstruction is presented, including error analysis. The method works by correlating the largest principal components of a matrix representation of the transport dynamics with a set of sparse measurements. The Lyapunov spectrum was measured and used to quantify the lifetime of each principal component. The method was tested on the $500 \mathrm{~K}$ isentropic surface with stratospheric ozone concentration measurements from the Polar Aerosol and Ozone Measurement (POAM) III satellite instrument during October and November 1998 and compared with the older proxy tracer method which works by correlating measurements with a single other tracer or proxy. Using a 60 day integration time and five (5) principal components, cross validation of globally reconstructed ozone and comparison with ozone sondes returned root-mean-square errors of $0.16 \mathrm{ppmv}$ and $0.36 \mathrm{ppmv}$, respectively. This compares favourably with the classic proxy tracer method in which a passive tracer equivalent latitude field was used for the proxy and which returned RMS errors of 0.22 ppmv and 0.59 ppmv for cross-validation and sonde validation respectively. The method seems especially effective for shorter lived tracers and was far more accurate than the classic method at predicting ozone concentration in the Southern hemisphere over the same time period. It is also more effective when reconstruction is performed over the entire Earth rather than a single hemisphere allowing for seamless reconstruction of global fields.
\end{abstract}

\section{Keywords}

transport dynamics, interpolation methods, satellite remote sensing, tracer advection, assimilation models, numerical analysis, transport modelling; ozone 


\section{Contents}

1 Introduction $\quad 2$

2 Theory 3

2.1 Tracer dynamics $\ldots \ldots \ldots \ldots \ldots$

2.2 Principal component proxy . . . . . . . . . . . . 4

2.3 Classic proxy tracer . . . . . . . . . . . . . . . . . 6

2.4 Lyapunov exponents . . . . . . . . . . . . . . . . . . . . 6

2.5 Special properties . . . . . . . . . . . . . .

2.6 Error analysis . . . . . . . . . . . . . . . . . . . . . . 8

3 Models and data 10

3.1 Tracer simulation . . . . . . . . . . . . . . . . . 10

3.2 Polar Ozone and Aerosol Measurement III instrument . . . . . . 10

3.3 National Center for Environmental Prediction reanalysis data . . 11

3.4 Ozone sonde data . . . . . . . . . . . . . . . . . . . 11

4 Numerical preliminaries $\quad 12$

4.1 Tracer correlation . . . . . . . . . . . . . . . . . 12

4.2 Calculating Lyapunov exponents . . . . . . . . . . . . . 13

5 Ozone reconstruction $\quad \mathbf{1 6}$

5.1 Cross-validation . . . . . . . . . . . . . . . . . . 16

5.2 Sonde validation $\ldots \ldots \ldots \ldots \ldots \ldots$

6 Discussion and conclusions 26

7 Acknowledgements 27

$\begin{array}{ll}\text { References } & \mathbf{2 7}\end{array}$

A Model properties 28

A.1 Mass conservation . . . . . . . . . . . . . 28

A.2 Diffusion and the Lyapunov spectrum . . . . . . . . . . 29 29

B Deviation from equal area 31

\section{Introduction}

Satellite remote sensing instruments have become invaluable for monitoring trace atmospheric constituents such as ozone. One area in which they frequently fall short is density of coverage. Short of comprehensive prognostic assimilation models, there are methods of interpolating sparse measurements that nonetheless take into account the atmospheric dynamics. One such method was first introduced in Butchart and Remsberg (1986) and used in Randall et al. (2002) to derive Northern hemisphere maps of ozone from Polar Ozone and Aerosol 
Measurement (POAM) III data. Proxy tracer analysis works by correlating measurements with another atmospheric variable, the proxy, that is conserved by the flow. The two most common proxies are potential vorticity (Hoskins et al., 1985) or a passive tracer simulated over a long time scale (Allen and Nakamura, 2003). In either case, it's standard practice to transform the proxy to an areaconserving Lagrangian coordinate called equivalent latitude before performing the reconstruction. This method is mainly useful for long-lived tracers such as ozone.

Here we introduce a new method of dynamical tracer reconstruction called principal component proxy analysis that is more appropriate for shorter-lived tracers because it works on shorter time scales. The method is summarized as follows: the passive tracer dynamics are represented as a matrix which linearly maps the initial tracer configuration to the final tracer configuration. We term this matrix the transport map. The transport map is decomposed using principal component analysis (PCA)-hence the name of the method-and the largest principal components are then fitted to the measurements. Because the transport map is integrated over only a short time scale and because it represents a large number of possible tracer configurations, with the most likely singled out through the decomposition, PC proxy should be better able to compensate for non-advective changes in concentration or sources and sinks.

\section{Theory}

\subsection{Tracer dynamics}

The advection-diffusion equation is given as follows:

$$
\frac{\partial q(\vec{x}, t)}{\partial t}=\{-\vec{v}(\vec{x}, t) \cdot \nabla+\nabla \cdot D \nabla\} q(\vec{x}, t)
$$

where $q$ is the tracer concentration, $\vec{x}$ is spatial position, $t$ is time, $\vec{v}$ is the fluid velocity, and $D$ is the diffusivity tensor. There is no mass-conservation term, $q \nabla \cdot \vec{v}$, either because $q$ is a volume-mixing ratio (vmr) or the fluid is incompressible $(\nabla \cdot \vec{v}=0)$. Sources are neglected since this would make the model nonlinear. See below.

In an Eulerian tracer simulation, the approximate value of $q$ is known only at discrete locations so can be represented as a vector, $\vec{q}=\left\{q_{i}\right\}$. The linear operator contained in the braces in Equation (1) is approximated as a matrix, $A$, which is multiplied with $\vec{q}$ :

$$
\frac{\mathrm{d} \vec{q}}{\mathrm{~d} t}=A(t) \vec{q}
$$

As an illustration, consider a second-order finite difference scheme in one dimension:

$$
\frac{\partial q_{i}}{\partial t}=\frac{v_{i}\left(q_{i-1}-q_{i+1}\right)}{2 \Delta x}+\frac{d\left(q_{i-1}+q_{i+1}-2 q_{i}\right)}{\Delta x^{2}}
$$




$$
=\left(\frac{v_{i}}{2 \Delta x}+\frac{d}{\Delta x^{2}}\right) q_{i-1}-\frac{2 d}{\Delta x^{2}} q_{i}+\left(-\frac{v_{i}}{2 \Delta x}+\frac{d}{\Delta x^{2}}\right) q_{i+1}
$$

where $d$ is a scalar diffusion coefficient, $\Delta x$ is the grid spacing and $v_{i}$ is the wind speed at the $i$ th grid point. Expressed as elements of the matrix, $A$ :

$$
\begin{aligned}
a_{i, i-1} & =\frac{v_{i}}{2 \Delta x}+\frac{d}{\Delta x^{2}} \\
a_{i, i} & =-\frac{2 d}{\Delta x^{2}} \\
a_{i, i+1} & =-\frac{v_{i}}{2 \Delta x}+\frac{d}{\Delta x^{2}}
\end{aligned}
$$

The actual transport model used in this study is described in Section 3.1.

To produce a general solution to Equation (2), we first substitute a matrix, $R$, for $\vec{q}$ :

$$
\frac{\mathrm{d} R\left(t_{0}, t\right)}{\mathrm{d} t}=A\left(t_{0}+t\right) R\left(t_{0}, t\right)
$$

We will call the matrix $R$ the discrete transport map or simply the transport map.

Unlike in most analyses, there are two parameters for the time: the integration start time, $t_{0}$, and the integration time, $t$. In this way, $R$ may be decomposed in terms of itself:

$$
R\left(t_{0}, t_{n}-t_{0}\right)=R\left(t_{n}, \Delta t_{n}\right) R\left(t_{n-1}, \Delta t_{n-1}\right) R\left(t_{n-2}, \Delta t_{n-2}\right) \ldots \quad R\left(t_{0}, \Delta t_{0}\right)
$$

where,

$$
t_{n}=t_{0}+\sum_{i=0}^{n} \Delta t_{i}
$$

It follows that $R(t, 0)=I$, for any $t$, where $I$ is the identity matrix.

Given $R$, we can calculate $\vec{q}$ given $\vec{q}$ at any other time:

$$
\vec{q}(t)=R\left(t_{0}, t-t_{0}\right) \vec{q}_{0}
$$

where $\vec{q}_{0}=\vec{q}\left(t_{0}\right)$ is the initial tracer configuration.

To make this solution fully analytical, although not realizable in practice, we solve $R$ for an infinitessimal integration time using linear algebra:

$$
\lim _{\Delta t \rightarrow 0} R(t, \Delta t)=\exp [\Delta t A(t)]
$$

where exp is the matrix generalization of the exponential function.

\subsection{Principal component proxy}

Suppose we decompose a given transport map using singular value decomposition:

$$
R\left(t_{0}, t_{n}-t_{0}\right)=U S V^{T}
$$


where $t_{n}-t_{0}$ is the integration time, $S$ is a diagonal matrix of singular values, and both $U$ and $V$ are orthogonal matrices:

$$
U^{T} U=V^{T} V=I
$$

$U$ is the matrix of left singular vectors and $V$ is the matrix of right singular vectors (Press et al., 1992). This method of matrix decomposition is also known as principal component analysis or PCA, hence the name of the interpolation technique. Singular vectors are increasingly being used in meteorology both to quantify the predictability of a forecast and to generate perturbations for ensemble forecasts (Tang et al., 2006).

A good way to think of it is as a set of orthogonal initial conditions-the right singular vectors, $\left\{\vec{v}^{(i)}\right\}$-which, when the tracer dynamics are applied, map onto a set of orthogonal final conditions-the left singular vectors, $\left\{\vec{u}^{(i)}\right\}$-that have grown by respective factors $\left\{s_{i}\right\}$ :

$$
R \vec{v}^{(i)}=s_{i} \vec{u}^{(i)}
$$

The superscript denotes the column number: this notation will be used throughout. Also, the term PC or principal component will be used as a synonym for left singular vector.

The singular values are all positive and by convention are arranged from largest to smallest:

$$
s_{i} \leq s_{i-1}
$$

The matrix can normally be reconstructed to a high degree of accuracy using only a few of the largest singular values and vectors. This also makes the problem tractable since a typical size for the transport map might be [(360 . $181) \times(360 \cdot 181)]=[65160 \times 65160]$.

To correlate a set of sparse measurements, $\left\{m_{i}\right\}$, with the top $k$ principal components, we first find interpolates at each measurement location in the left singular vectors and then find a set of coefficients, $\vec{c}$, that minimizes the meansquare error of the interpolates versus the measurements. In any real problem, the measurements are unlikely to occur at the same time, so rather than using the left singular vectors, we use $R$ to advance the right singular vectors to the same time as the measurement. The time period during which measurements are admitted is the measurement window. While the difference between the integration start time, $t_{0}$, and the center of the measurement window is the lead time.

We have the following minimization problem:

$$
\min _{\vec{c}} \sum_{i}\left\{\sum_{j=1}^{k} c_{j} \vec{w}_{i} R\left(t_{0}, t^{(i)}-t_{0}\right) \vec{v}^{(j)}-m_{i}\right\}^{2}
$$

where $t^{(i)}$ is the time stamp of the $i$ th measurement, $m_{i}$, and $\vec{w}_{i}$ is a vector of interpolation coefficients. In this work, bilinear interpolation is used to interpolate measurement locations. Since $R$ is only known at discrete time values, $R\left(t_{0}, t^{(i)}-t_{0}\right) \vec{v}^{(j)}$ is also approximated through linear interpolation. 
Once the coefficients have been fitted, the tracer is reconstructed as follows:

$$
\vec{q}\left(t_{n}\right) \approx \sum_{i=1}^{k} c_{i} \vec{u}^{(i)}
$$

\subsection{Classic proxy tracer}

Contrast the above description of principal component proxy tracer analysis with the original proxy tracer technique, hereafter referred to as "classic" proxy tracer. In the earlier method, the measurements are simply correlated with another tracer (the proxy): either a passive tracer that has been advected continuously with periodic re-normalization (Allen and Nakamura, 2003) or some other quantity that is conserved by the flow such as potential vorticity (Randall et al., 2002; Hoskins et al., 1985). The regression is typically done to second-order and the proxy variable converted to an area-based "Lagrangian" coordinate called equivalent latitude (Butchart and Remsberg, 1986).

If $\vec{\Phi}=\left\{\Phi_{i}\right\}$ is the proxy field, then we have the following minimization problem:

$$
\min _{\vec{c}^{\prime}} \sum_{i}\left\{\sum_{j=0}^{N} c_{j}^{\prime}\left[\vec{w}_{i} \cdot \vec{\Phi}\left(t_{i}\right)\right]^{j}-m_{i}\right\}^{2}
$$

where $\vec{c}^{\prime}$ are the regression coefficients and $N$ is the order of the method. The tracer is reconstructed:

$$
q_{i}(t) \approx \sum_{j=0}^{N} c_{j}^{\prime} \Phi_{i}^{j}(t)
$$

where $i$ in this case runs from 1 to the number of grid points in the proxy field.

\subsection{Lyapunov exponents}

Suppose we have a system of ordinary differential equations (ODEs):

$$
\frac{\mathrm{d} \vec{r}}{\mathrm{~d} t}=\vec{f}(\vec{r}, t)
$$

where $\vec{r}$ is a vector of dependent variables. Linearize this about $\vec{r}$ using the tangent vector, $\nabla_{\vec{r}} f$ :

$$
\begin{aligned}
\frac{\mathrm{d}}{\mathrm{d} t}(\vec{r}+\delta \vec{r}) & \approx \vec{f}+\nabla_{\vec{r}} \vec{f} \cdot \delta \vec{x} \\
\frac{\mathrm{d}}{\mathrm{d} t} \delta \vec{r} & \approx \nabla_{\vec{r}} \vec{f} \cdot \vec{r}
\end{aligned}
$$

where $\delta \vec{r}$ is a vector of infinitessimal error vectors. Now define the tangent model, $H$, such that:

$$
\frac{\mathrm{d}}{\mathrm{d} t} H=\nabla_{\vec{r}} \vec{f} H
$$


A passive Eulerian tracer simulation is linear: taking Equation (21) as our system of ODEs in (18) then setting $\vec{r}=\vec{q}$, we have, $\vec{f}(\vec{q}, t)=A(t) \vec{q}$, while the tangent vector is given as:

$$
\nabla_{\vec{q}} \vec{f}=A
$$

Hence the transport matrix, $R$, is equivalent to the tangent model, $H$.

The Lyapunov exponents are defined as the logarithms of the time averages of the singular values in the limit as time goes to infinity:

$$
\lambda_{i}=\lim _{t \rightarrow \infty} \frac{1}{t} \log s_{i} ; \quad \lambda_{i-1} \leq \lambda_{i}
$$

where $s_{i}$ is the $i$ th singular value $(\mathrm{Ott}, 1993)$. For most systems:

$$
|\delta \vec{r}| \approx|\delta \vec{r}(0)| \exp \left(\lambda_{i} t\right)
$$

That is, as $H$ is integrated forward, the largest singular value and the largest singular vector will increasingly begin to dominate (Ott, 1993). The Lyapunov exponents can help us gauge the significance of each singular vector at a given lead time.

\subsection{Special properties}

An important property of flow tracers is that the amount of substance is conserved:

$$
\sum_{i} q_{i}=\text { const. }
$$

The equation is exact if the simulation uses an equal area grid and the fluid is incompressible $(\nabla \cdot \vec{v}=0)$. From this it follows:

$$
\begin{aligned}
& \sum_{i} r_{i j}=1 \\
& \sum_{i} a_{i j}=0
\end{aligned}
$$

See Appendix A.1 for the derivation.

All gridded Eulerian tracer simulations are by necessity diffusive. Given in addition the constraint above in (25), we can also show that all the singular values are less-than-or-equal-to one:

$$
0 \leq s_{i} \leq 1
$$

thus the Lyapunov exponents in turn will all be negative or zero. Section 4.2 provides a numerical demonstration while Appendix A.2 gives the derivation. 


\subsection{Error analysis}

A detailed error analysis can help us both to better understand the technique and to chose the best parameters for a given interpolation. Real flow tracers will have sources and sinks, thus we introduce a source term, $\sigma$, to Equations (6) and (8):

$$
\begin{aligned}
\vec{q}\left(t_{n}\right) \approx & \vec{\sigma}_{n}+ \\
& R\left(t_{n-1}, \Delta t_{n-1}\right)\left[\vec{\sigma}_{n-1}+\right. \\
& R\left(t_{n-2}, \Delta t_{n-2}\right)\left[\vec{\sigma}_{n-2}+\right. \\
& \vdots \\
& +R\left(t_{1}, \Delta t_{1}\right)\left[\vec{\sigma}_{1}+\right. \\
& \left.\left.\left.R\left(t_{0}, \Delta t_{0}\right) \vec{q}_{0}\right] \ldots\right]\right]
\end{aligned}
$$

where $\vec{\sigma}_{i}$ is the integrated source term for the $i$ th time step. Expanding:

$$
\vec{q}\left(t_{n}\right)=R\left(t_{0}, t_{n}-t_{0}\right) \vec{q}_{0}+\sum_{i=1}^{n} R\left(t_{i}, t_{n}-t_{i}\right) \vec{\sigma}_{i}
$$

To get a handle on the error, we consider first a fully passive tracer (no sources or sinks) started with initial conditions $\vec{q}_{0}$. Expanding this in terms of the right singular vectors with a set of coefficients, $\vec{c}_{0}$ :

$$
\vec{q}_{0}=V \vec{c}_{0}
$$

means that the final tracer takes the following form:

$$
\vec{q}\left(t_{n}\right)=U S \vec{c}_{0}
$$

however we are only calculating the top $k$ singular vectors, so the interpolation looks like this:

$$
\vec{q}\left(t_{n}\right) \approx \sum_{i=0}^{k} c_{0 i} s_{i} \vec{u}^{(i)}
$$

The most significant source of error are the terms left out of the equation. The smaller the remaining singular values, $\left\{s_{i} \mid i=\left[k+1 . . n_{p}\right]\right\}$, the smaller the error, where $n_{p}$ is the total number of grid points in the tracer. This is why the Lyapunov exponents are useful: they tell us how fast the singular values shrink.

A tracer interpolated with all the singular vectors will take the form:

$$
\vec{q}\left(t_{n}\right)=U \vec{c}
$$

Substitution of (31) into (30) and comparison with (34) produces the following:

$$
\vec{c}=S \vec{c}_{0}+U^{T} \sum_{i=1}^{n} R\left(t_{i}, t_{n}-t_{i}\right) \vec{\sigma}_{i}
$$


In other words, we need to project the integrated source terms onto the singular vectors. As before, the error is given by terms not included in the analysis:

$$
\vec{\epsilon}=\sum_{i=k+1}^{n_{p}} \vec{u}^{(i)}\left[s_{i} c_{0 i}+\vec{u}^{(i)} \cdot \sum_{j=1}^{n} R\left(t_{j}, t_{n}-t_{j}\right) \vec{\sigma}_{j}\right]
$$

Note that the first occurrence of the singular vector, $\vec{u}^{(i)}$, does not cancel out the second occurrence because the factor in square brackets is a scalar, i.e., order of operations matters.

The first term in Equation (36) sums the components of the initial tracer configuration that project onto the smaller singular vectors not included in the analysis. This term will be small because of the shrinking of singular values over time. The second term are the components of the source terms projected onto the same singular vectors. The less the source terms line up with the largest singular vectors, the larger this source of error.

Equation (36) suggests two approaches to reducing the error. The first is to reduce the size of $R\left(t_{j}, t_{n}-t_{j}\right)$ so that the source terms grow as little as possible. This would suggest that the measurement window should be in the middle of the integration, i.e. the lead time is half the integration time. The second is to make this factor as close as possible to $R\left(t_{n}, t_{n}-t_{0}\right)$ so that projection onto the singular vectors leaves the term $S V$ and the leftover smaller components are shrunk by the singular values. This would suggest that the measurement window should be towards the end of the integration, i.e. the lead time is the same as the integration time.

To understand this last point, rewrite Equation (36) as follows:

$$
\vec{\epsilon}=\sum_{i=k+1}^{n_{p}} s_{i} \vec{u}^{(i)}\left[c_{0 i}+\vec{v}^{(i)} \cdot \sum_{j=1}^{n} R^{-1}\left(t_{0}, t_{j}-t_{0}\right) \vec{\sigma}_{j}\right]
$$

Note that because of diffusion, a backwards integration is not equivalent to the inverse of the forwards integration, but only approximately so, that is, $R(t+$ $\Delta t,-\Delta t) \approx R^{-1}(t, \Delta t)$, with the approximation becoming worse as $\Delta t$ becomes larger.

Measurement error and fitting discrepancies can be treated in the same way as any other linear least squares problem. It stands to reason that having fewer measurements will magnify both measurement errors and discrepancies generated by sources and sinks. More measurements will allow the use of more singular vectors, reducing both the error terms in (36). In this paper we will take an empirical approach to the error analysis by validating reconstructed tracer fields against actual measurements whenever possible. 


\section{Models and data}

\subsection{Tracer simulation}

To generate the transport maps, the ctraj software package is used (http://ctraj.sf.net) (Mills, 2004, 2009). The software is written in $\mathrm{C}++$ and contain programs for gridded, two-dimensional, semi-Lagrangian tracer advection on an azimuthallyequidistant-projected coordinate system:

$$
\begin{aligned}
& x=r \cos \theta \\
& y=r \sin \theta \\
& r=R_{E}(\pi / 2-h \phi)
\end{aligned}
$$

where $\theta$ is longitude, $\phi$ is latitude and $h$ is the hemisphere in which the projection is defined:

$$
h=\left\{\begin{aligned}
1 ; & \text { North } \\
-1 ; & \text { South }
\end{aligned}\right.
$$

The resulting space has the following metric coefficients:

$$
\begin{aligned}
& \left(\frac{\mathrm{d} s}{\mathrm{~d} x}\right)^{2}=\frac{1}{r^{2}}\left[\frac{R_{E}^{2}}{r^{2}} \sin ^{2}\left(\frac{r}{R_{E}}\right) y^{2}+x^{2}\right] \\
& \left(\frac{\mathrm{d} s}{\mathrm{~d} y}\right)^{2}=\frac{1}{r^{2}}\left[\frac{R_{E}^{2}}{r^{2}} \sin ^{2}\left(\frac{r}{R_{E}}\right) x^{2}+y^{2}\right]
\end{aligned}
$$

where $R_{E}$ is the radius of the Earth. Two fields are advected simultaneously: one for the Northern hemisphere $(h=1)$ and one for the Southern hemisphere $(h=-1)$, with equatorial crossings accounted for.

Because it is a semi-Lagrangian simulation, the factors, $\left\{R\left(t_{i}, \Delta t\right)\right\}$, are output directly as sparse matrices by calculating the interpolation coefficients. By storing the output as sparse matrices and not multiplying them through until needed, it becomes possible to calculate the singular values and vectors through iterative methods such as the Lanczoz method (Golub and van Loan, 1996). The Arnoldi package (ARPACK) (Lehouca and Scott, 1996) is used to compute the eigenvalues and eigenvectors needed for the SVD.

To calculate back-trajectories, a fourth-order Runge-Kutta integration scheme was used with a 1.2 hour time-step. Back trajectories were linearly interpolated after each 1-day, Eulerian time step. Gridding in both hemispheres is 50 by 50 , or 400km-, 3.6-degree-latitude-separation at the pole.

\subsection{Polar Ozone and Aerosol Measurement III instrument}

The Polar Ozone and Aerosol Measurement (POAM) III instrument was a solaroccultation instrument mounted on the SPOT-4 sun-synchronous, low-earthorbit satellite (Lucke et al., 1999). Operating between March 1998 and December 2005, it had nine channels in the visual and near infrared range. Using optimal estimation (Rodgers, 2000), ozone profiles have been retrieved within a 


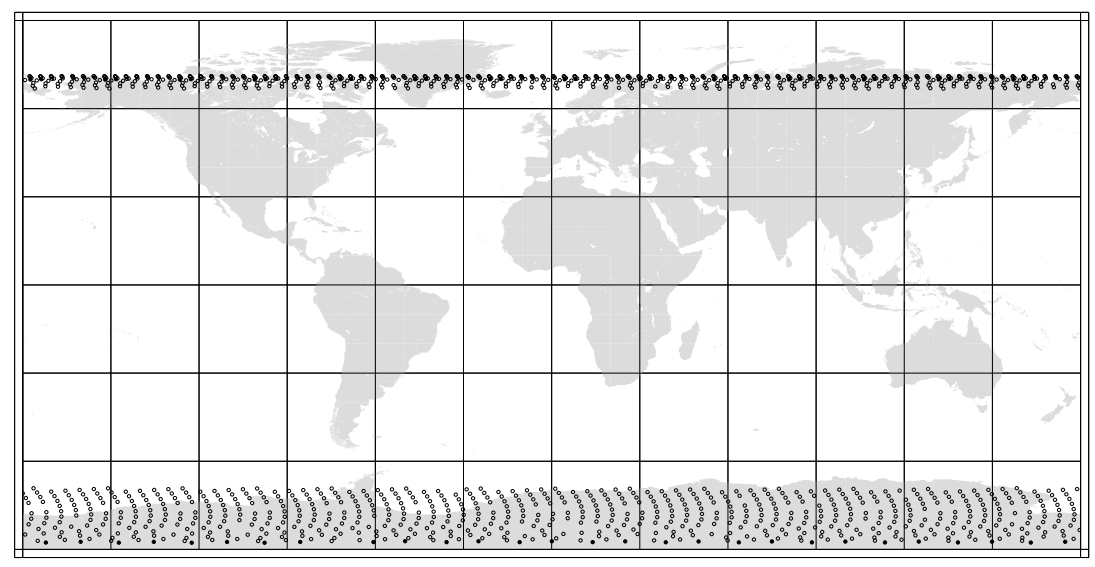

Figure 1: Locations of all POAM III measurements used in this study between Sept. 25, 1998 and November 18, 1998. Highlighted points are for the first time grid (two days worth of data) between Sept. 25, 1998 0:00 and Sept 27, 1998 0:00 UTC.

pair of narrow latitude bands in both the Arctic and Antarctic (Lumpe et al., 2002). The instrument is capable of returning 28 or 29 measurements per day, alternating between Northern and Southern hemisphere, however because of a malfunction in the instrument, it normally operates in only one or the other hemisphere for longer periods. Therefore, we confine ourselves to earlier data, October and November 1998, when more frequent and diverse measurements are available. The locations of all POAM measurements used in this study are plotted in Figure 1

\subsection{National Center for Environmental Prediction reanal- ysis data}

The National Center for Environmental Prediction (NCEP) supplies, free-ofcharge, gridded ( 2.5 by 2.5 degrees longitude/latitude, 4 time daily), reanalyzed climate data starting in 1948 (Kalnay et al., 1996). Daily averaged wind and temperature data was used to drive the advection model.

\subsection{Ozone sonde data}

The World Ozone and Ultraviolet Data Centre (WOUDC) collects ozone sonde data from around the world (Hare et al., 2000). A list of all contributors is available on the website: http://woudc.org. The data is archived by Environment Canada. The location of all the launch stations used in the validation exercises is shown in Figure 2, Sonde locations are a good match for the POAM III measurement locations since they are primarily at high latitudes with few stations towards the equator. 


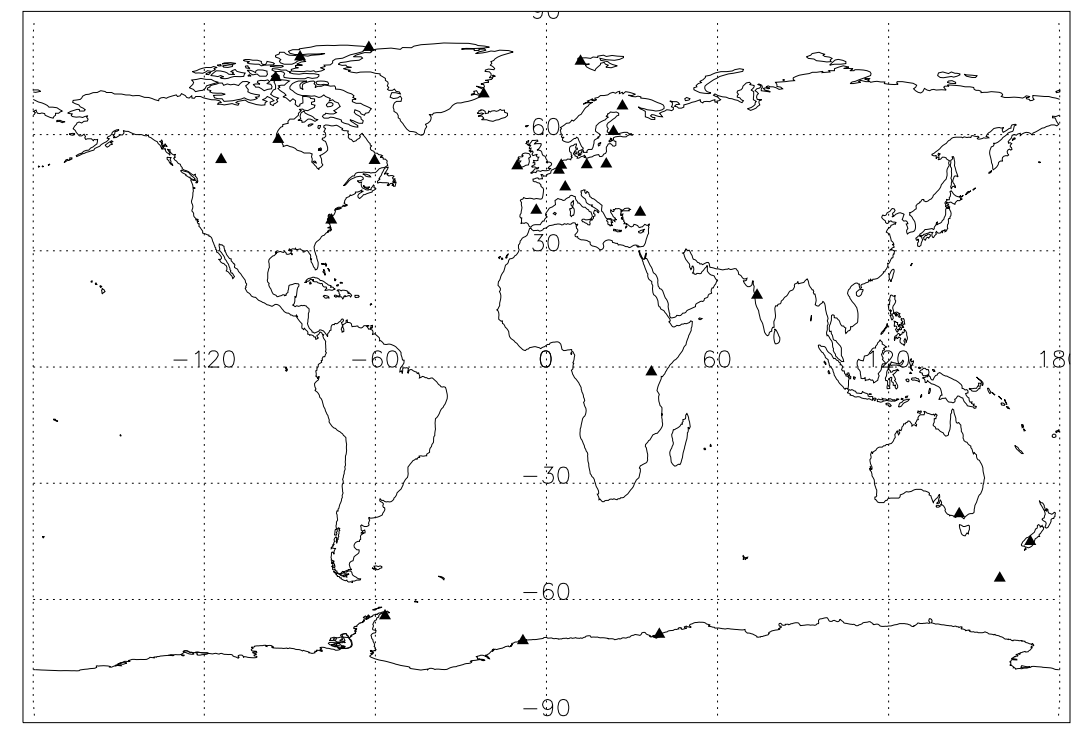

Figure 2: Locations of ozone sonde launch stations

\section{Numerical preliminaries}

For reference, examples of singular vectors derived from the transport map, $R(t, \Delta t)$, where $t$ is August 1, 1998 and $\Delta t$ is sixty days, are shown in Figure 3. If $R(t, \Delta t)=U S V^{T}$, Figure 3a. shows the first right singular vector, $\vec{v}^{(1)}$, while Figure $3 \mathrm{~b}$. shows the corresponding left singular vector, $\vec{u}^{(1)}$; Figure 3. shows the second right singular vector, $\vec{v}^{(2)}$, while Figure 3 b. shows the corresponding left singular vector, $\vec{u}^{(2)}$ and so on. While the globally projected fields representing the right singular vectors appear to be quite similar and are indeed quite strongly correlated, the abstract vectors from which they are derived have negligible dot products.

\subsection{Tracer correlation}

Two differently-initialized tracers, when integrated with the same wind fields over a long time period, become correlated. As discussed in Section 2.3, this can be used to infer global fields of a long-lived tracer such as ozone based on only a few sparse measurements (Allen and Nakamura, 2003; Randall et al., 2002). Figure 4 demonstrates this with the extreme example of an initially zonallysymmetric tracer and an initially meridionally-symmetric, two-dimensional tracer. Tracers are passively advected with National Center for Environmental Prediction (NCEP) reanalysis 1 data at the $500 \mathrm{~K}$ isentrop (Kalnav et al., 1996). The Pearson coefficient, weighted by grid size, is applied over the whole field at a 


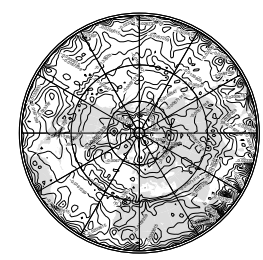

a. Right SV 1

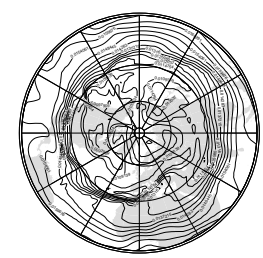

b. Left SV 1

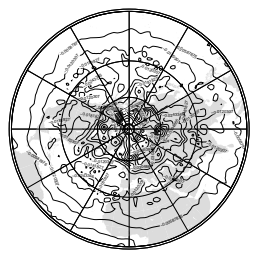

c. Right SV 2

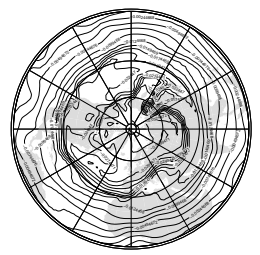

d. Left SV 2

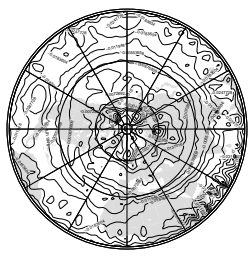

e. Right SV 3

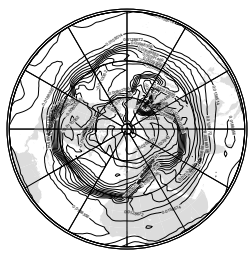

f. Left SV 3

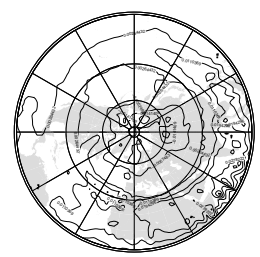

g. Right SV 4

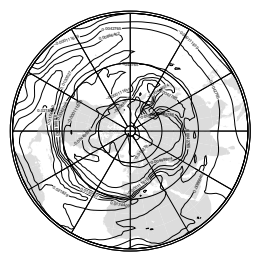

h. Left SV 4

Figure 3: An example of the singular vectors derived from a discrete transport map. Sixty day integration started on August 11998 on the $500 \mathrm{~K}$ isentrop driven by NCEP wind fields.

single time-step.

We also plot the Pearson correlation coefficient of the first tracer with the largest singular vector. We see that, because of Equation (24), they too become correlated over time. This at least partially explains the efficacy of the proxy tracer method. The periodic dips in correlation are not yet understood. A sample PC as compared with the tracer is shown in Figure 5

\subsection{Calculating Lyapunov exponents}

Figure 6 plots the time evolution of the singular values. From this we can calculate the Lyapunov spectrum by making straight line fits of their logarithms. While the resulting fields may develop into complex fractals (Mills, 2009) the Lyapunov spectrum shows that the tracer dynamics themselves are not truly chaotic (in fact cannot be), but are only on the cusp: the largest singular value remains approximately constant. It also shows how quickly the other singular vectors decay, so that the largest will eventually dominate in accordance with Equation (24).

All the Lyapunov exponents are less than zero, with the largest roughly zero, in agreement with the inequality in (28), even though Equation (25) is true only approximately. In this case it is close enough: the smallest boxes on the azimuthal equidistant grid will deviate from the largest by a factor of only $2 / \pi^{2}+1 / 2 \approx 0.7$ (see derivation in Appendix $B$ ) while most real fluids are approximately non-divergent, especially when considered over long time scales. 


\section{Tracer correlation}

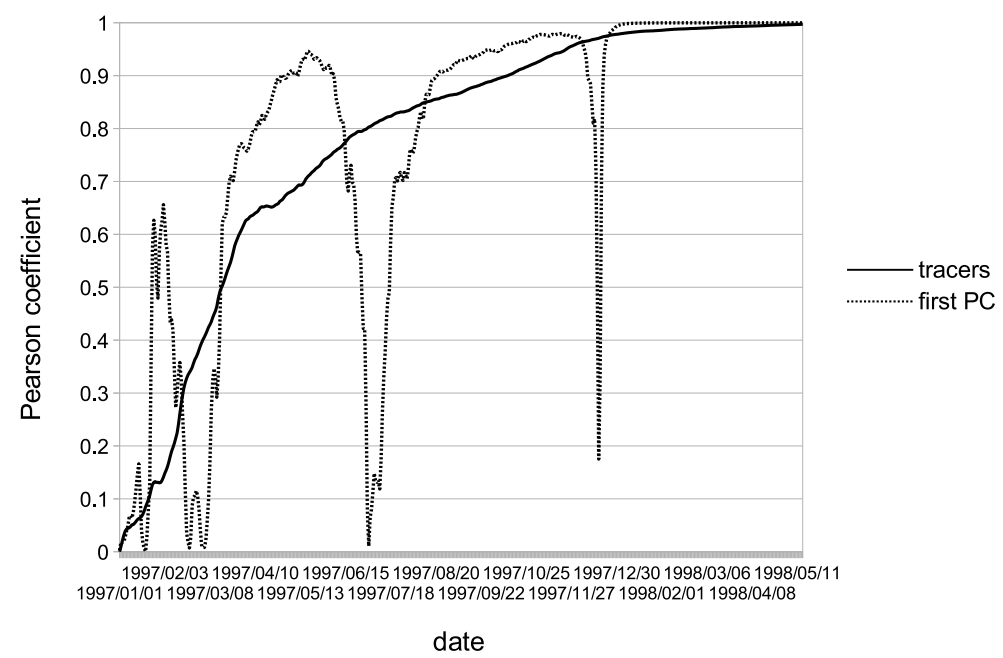

Figure 4: The Pearson correlation coefficient over time of two differentlyinitialized, two-dimensional tracers (broken line)-zonally symmetric and meridionally symmetric-and of the zonally-symmetric-initialized tracer with the first principal component. The simulation was driven with NCEP reanalysis 1 data on the $500 \mathrm{~K}$ isentropic level with an Eulerian time-step of 20 hours and a Lagrangian time-step of one 1.2 hours. 
a. Tracer

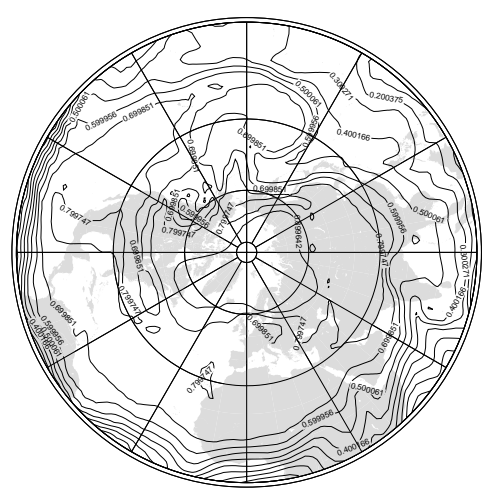

b. First PC

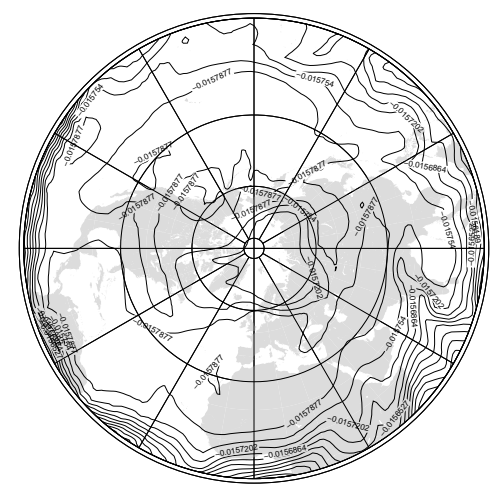

Figure 5: Comparison of a simulated tracer (a.) and the first principal component (b.) for the same time integration. Integration started on January 1, 1997 and continued until December 1, 1997, a period of 334 days and was performed on the $500 \mathrm{~K}$ isentrop with NCEP wind fields.

\section{Lyapunov spectrum}

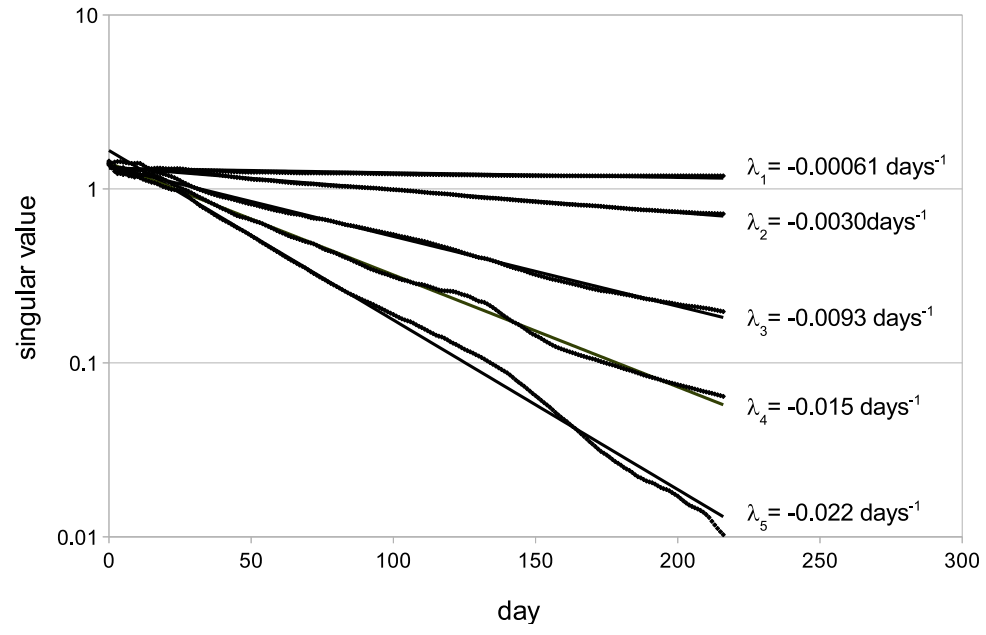

Figure 6: Plot of the top five (5) singular-values of a semi-Lagrangian tracer simulation over time. Straight-line fits return the Lyapunov-spectrum. The simulation was done on the $500 \mathrm{~K}$ isentropic level. 


\section{Ozone reconstruction}

In this section we use $\mathrm{PC}$ proxy to reconstruct two-dimensional ozone fields from POAM III satellite data. To perform the analysis, we need to choose an integration time, $t_{n}-t_{0}$, in Equation (10), as well as a measurement window. The integration time determines how long the tracer is advected before performing the SVD. Measurements are selected from within the measurement window. We also need to choose a lead time which determines how far from the beginning of the integration, $t_{0}$, the measurement window is centered. For this experiment, it was centered at the end of the integration period. Placing the measurement window in the middle of the integration period was found to work poorly. The Lyapunov spectrum can help us select the number of singular vectors as it shows how many remain significant at a given lead time-see Figure 6.

Ozone fields were reconstructed daily on the $500 \mathrm{~K}$ isentrop between September 26 and November 17, 1998, which is one of only a few periods in which POAM III was operating in both hemispheres simultaneously. The tracer simulation was run at a 50 by 50 resolution or 400 by $400 \mathrm{~km}$ at the poles with a 1.2 hour Runge-Kutta time step for the back-trajectories and a 1 day Eulerian time step. The integration time was 60 days with the same lead time and five principal components were used unless otherwise noted. The measurement window was two days.

An example of a reconstructed ozone field is shown in Figure 7 with Figure $7 \mathrm{~h}$. using the classic proxy tracer method while Figure $7 \mathrm{~b}$. uses the PC proxy method. Because POAM III data is confined to two rather narrow latitude bands near the poles, values near the equator may not be that accurate. Nonetheless, the author thought it important to test the method with global reconstructions to see how well the PC proxy method can extrapolate to areas where measurements are sparse or non-existant. As we will see, PC proxy is not only more accurate than the classic method, it can also be better at taking into account non-local information when it does not become unstable due to over-fitting.

\subsection{Cross-validation}

In the first validation exercise, the POAM data was randomly separated into two equal groups, each of which was used to predict the other. Since the POAM measurements are closely grouped, falling into one of two narrow latitude bands in the Arctic and Antarctic, and spaced at roughly 85 degrees longitude between consecutive measurements, skill scores will tend to be quite high. PC proxy was compared to a classic proxy tracer with a second order fit. The same tracer simulation as used for PC proxy was used to generate equivalent latitude fields (Allen and Nakamura, 2003) for the classic proxy tracer but with a two year spin-up. Unlike in Randall et al. (2002), the reconstruction was done over the entire globe. Reconstructed ozone fields were linearly interpolated to match the locations of the sonde measured test group.

Figure 8 shows the cross-validation results for classic proxy tracer while 


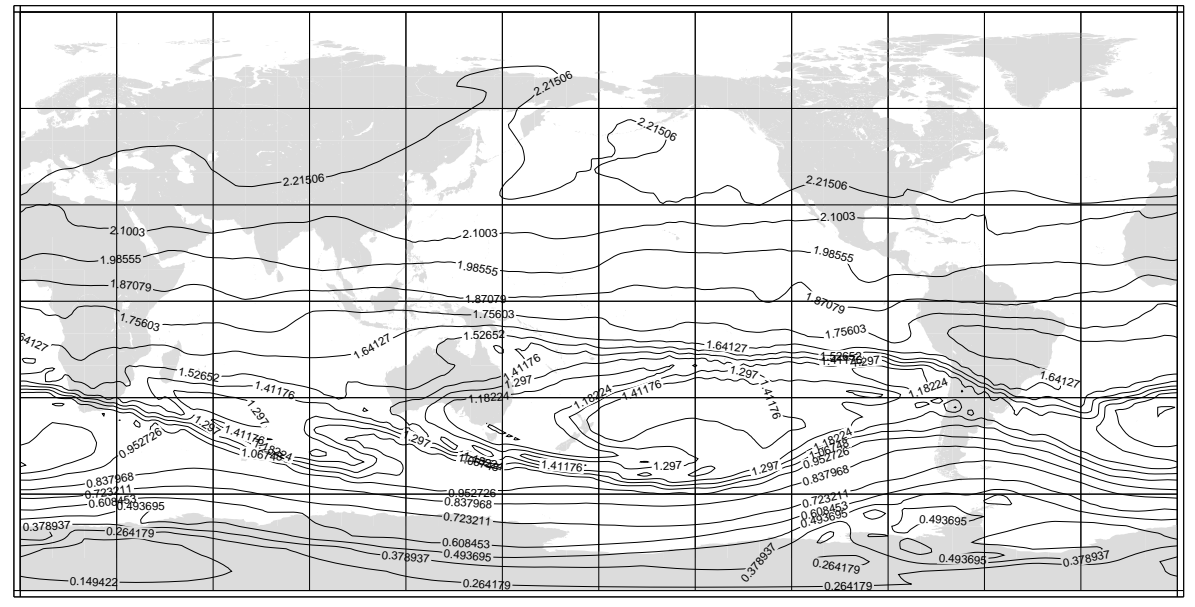

a. Classic proxy tracer

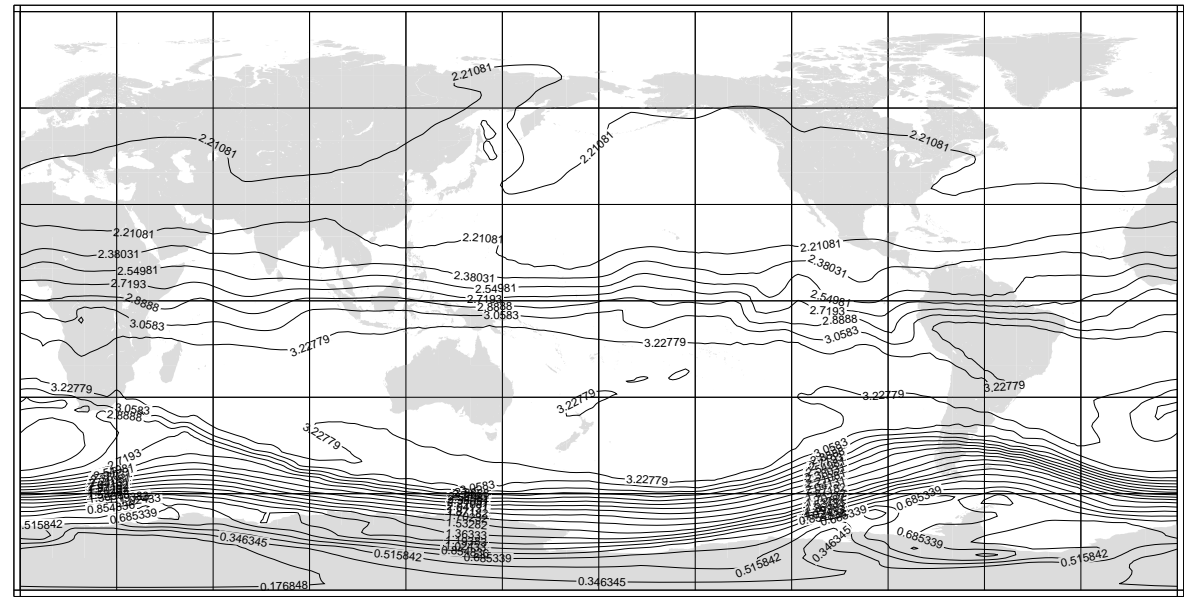

\section{b. PC proxy}

Figure 7: Sample globally reconstructed ozone fields on the $500 \mathrm{~K}$ isentrop for October 1, 1998. Units are parts-per-million by volume (ppmv). 


\section{a. Proxy tracer cross-validation}

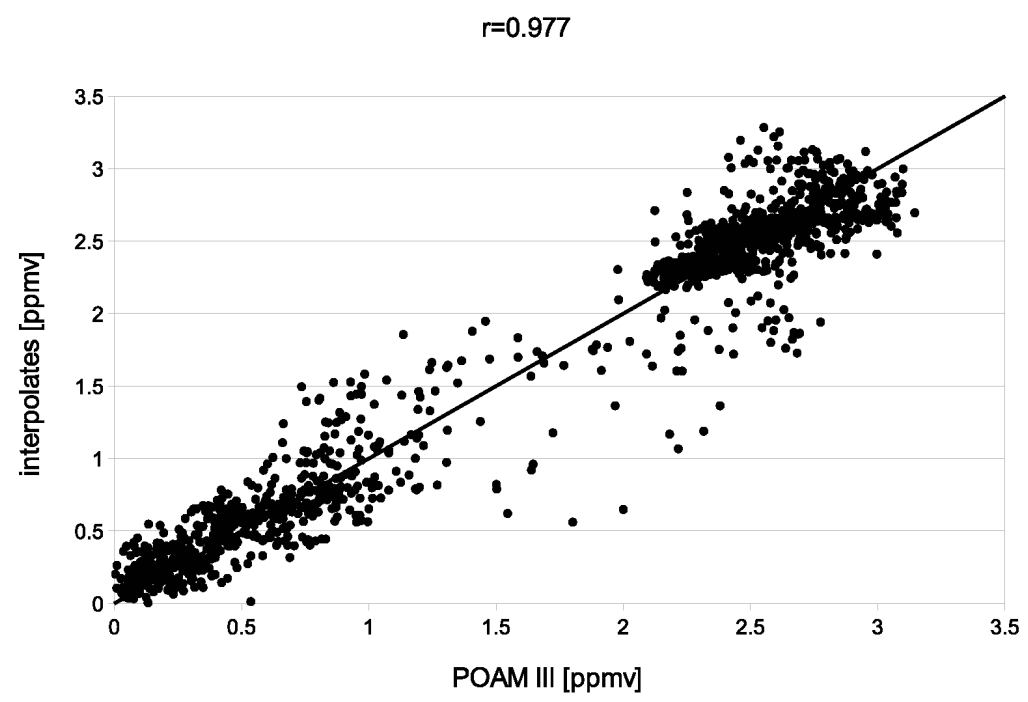

b. Error statistics

$\mu=-0.023 ; \sigma=1.18$

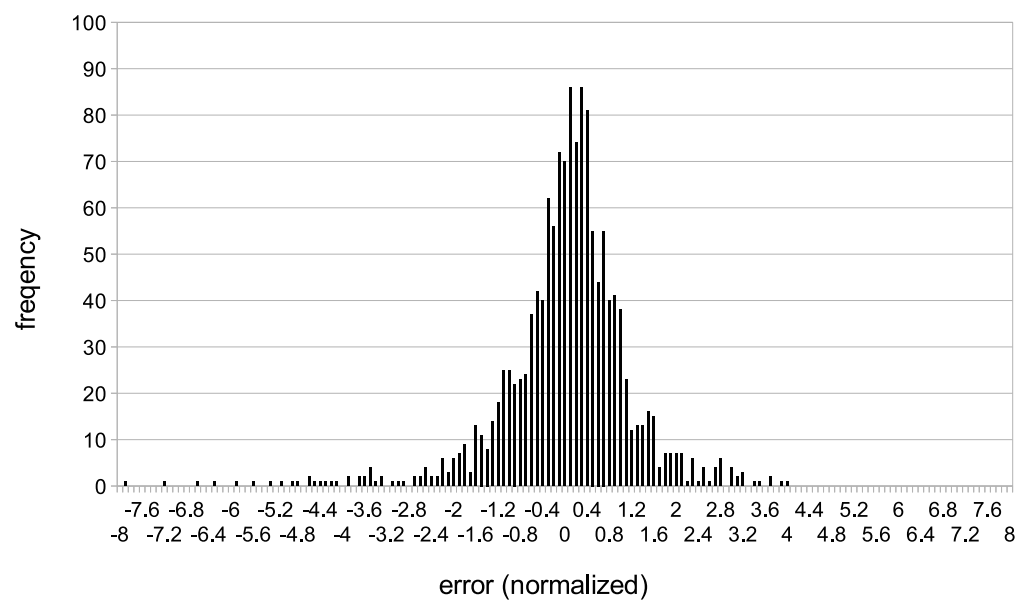

Figure 8: Cross validation of classic second-order proxy tracer ozone reconstruction. The reconstruction was done globally on the $500 \mathrm{~K}$ isentrop from POAM III data between September 26, 1998 and November 17, 1998 using a measurement window of 2 days. 


\section{a. PC proxy cross-validation}
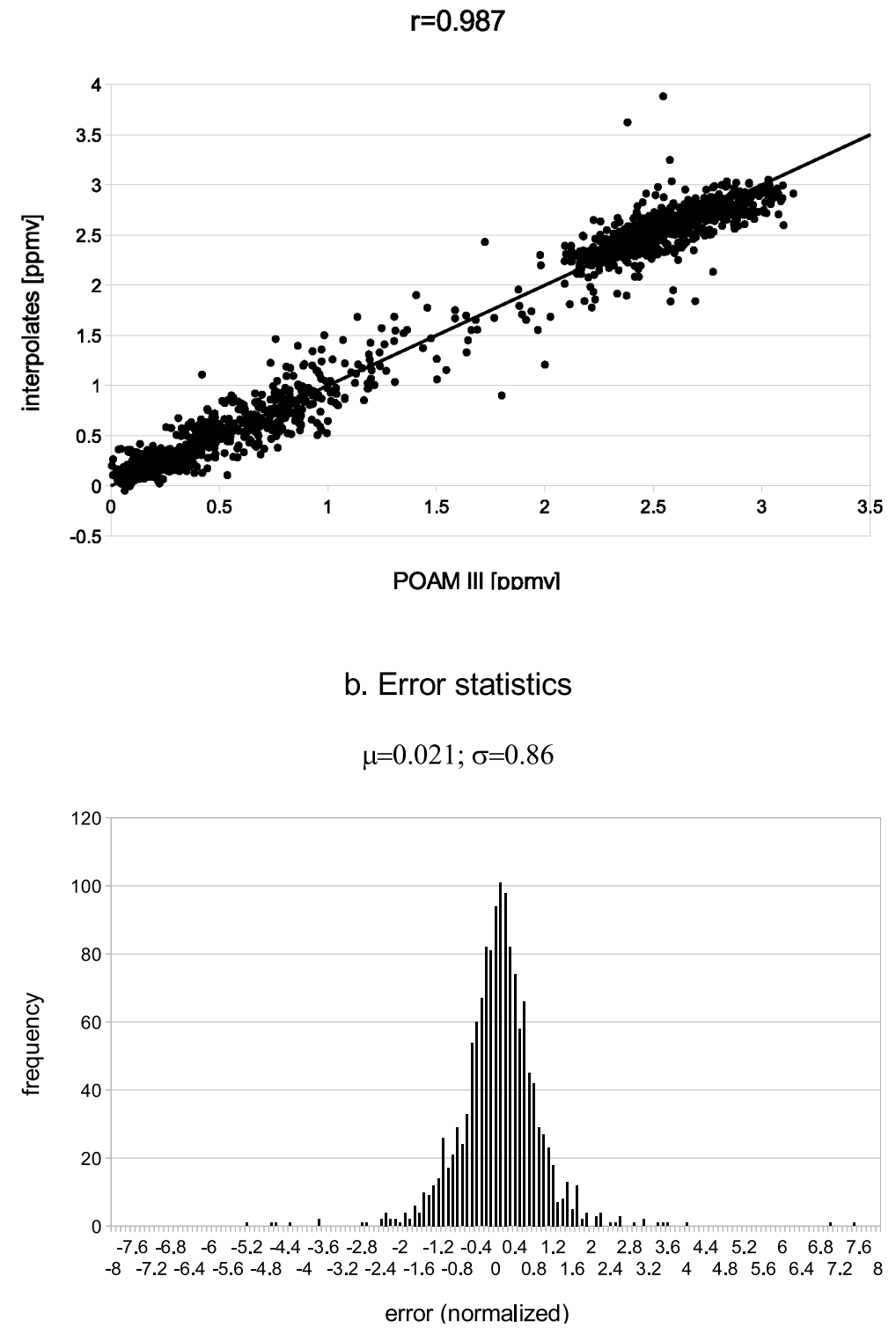

Figure 9: Cross validation of principal component proxy ozone reconstruction. The reconstruction was done globally on the $500 \mathrm{~K}$ isentrop from POAM III data between September 26, 1998 and November 17, 1998 using 5 principal components, an integration time of 60 days, and a measurement window of 2 days. 


\section{a. Proxy tracer}

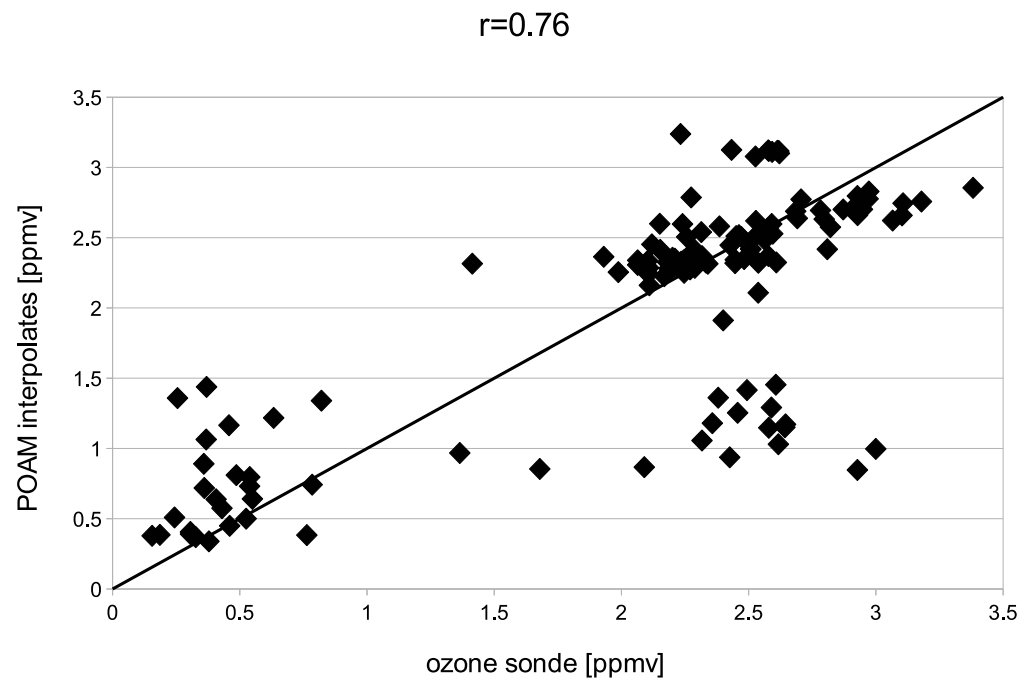

b. Error statistics

$\mu=-0.074 ; \sigma=0.59$

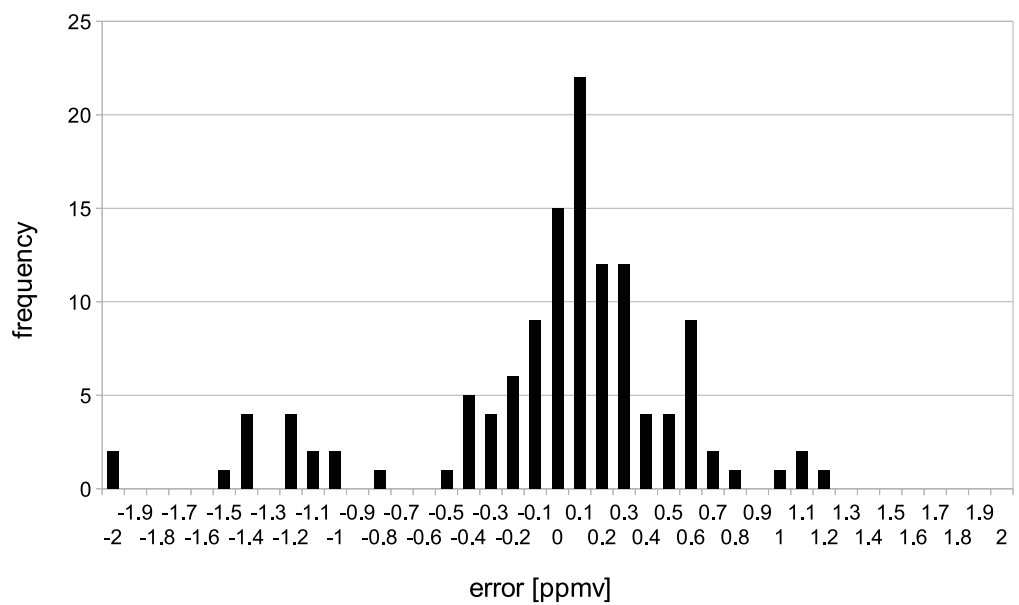

Figure 10: Validation of classic second-order proxy tracer ozone reconstruction against ozone sonde measurements. The econstruction was done globally on the $500 \mathrm{~K}$ isentrop from POAM III data between September 26, 1998 and November 17, 1998 using a measurement window of 2 days. 
a. PC proxy

$r=0.91$

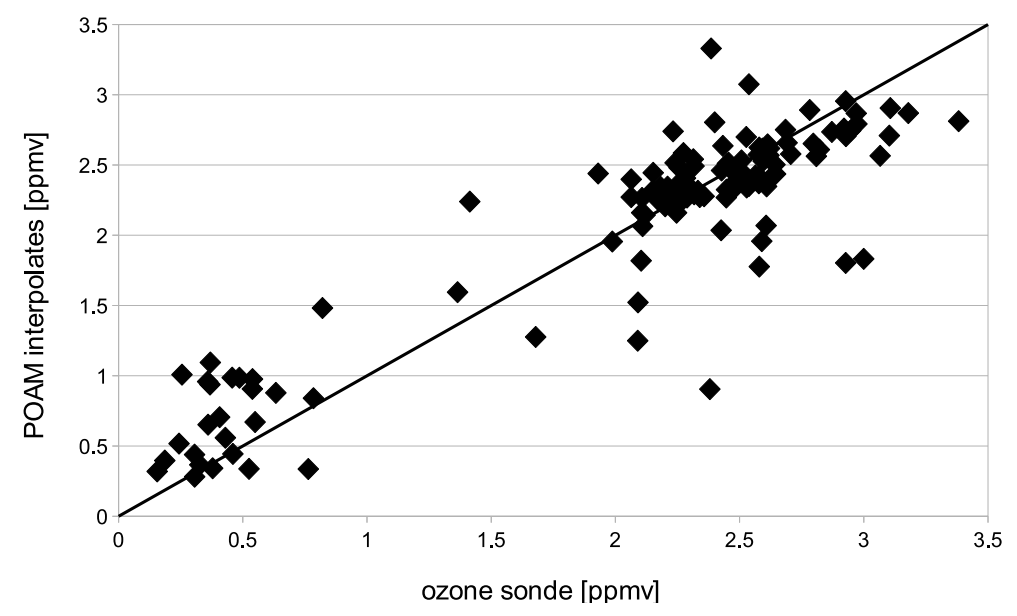

b. Error statistics

$\mu=-0.0040 ; \sigma=0.36$

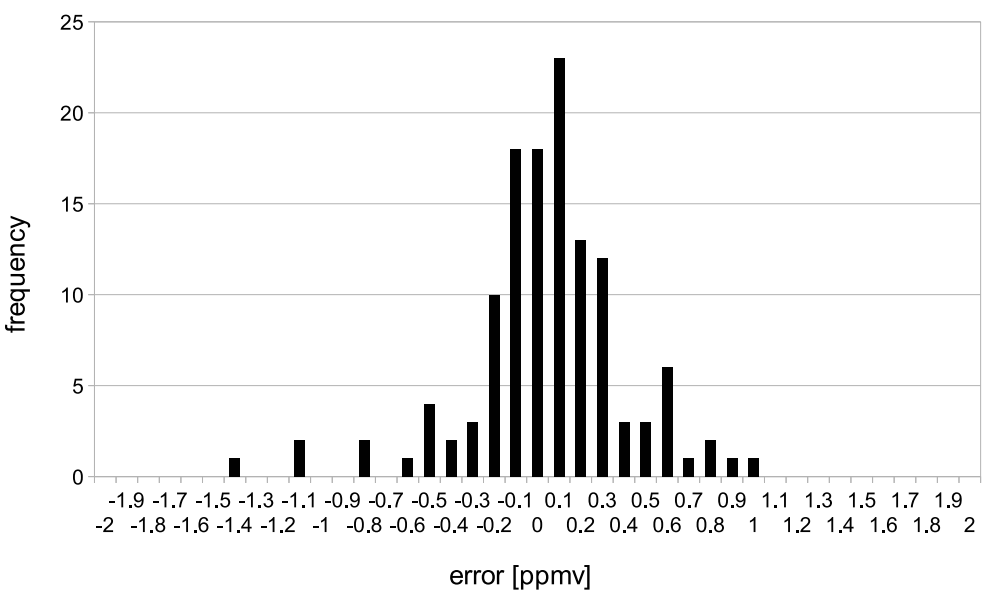

Figure 11: Validation of principal component proxy ozone reconstruction against ozone sonde measurements. The reconstruction was done globally on the 500 K isentrop from POAM III data between September 26, 1998 and November 17, 1998 using 5 principal components, an integration time of 60 days, and a measurement window of 2 days. 
Table 1: Summary of ozone sonde trials. $h_{1}$ is the hemisphere over which the reconstruction was done. $h_{2}$ is the hemisphere for which skill scores are shown. -1 indicates Southern, +1 Northern and 0 the whole globe. FAC2 is fraction of estimates within a factor of 2 .

\begin{tabular}{|llll|lll|lll|}
\hline method & $k \vee N$ & $h_{1}$ & $h_{2}$ & $r$ & $\mu$ & $\sigma$ & $\mu / \sigma_{q}$ & $\sigma / \sigma_{q}$ & FAC2 \\
\hline \hline Classic & 2 & 0 & 0 & 0.759 & -0.0742 & 0.592 & -0.0857 & 0.683 & 0.857 \\
PC & 5 & 0 & 0 & 0.909 & -0.00396 & 0.362 & -0.00456 & 0.417 & 0.913 \\
\hline Classic & 2 & 0 & +1 & 0.529 & 0.0782 & 0.296 & 0.235 & 0.891 & 1. \\
PC & 5 & 0 & +1 & 0.748 & -0.0138 & 0.221 & -0.0415 & 0.664 & 1. \\
\hline Classic & 2 & 0 & -1 & 0.590 & -0.349 & 0.846 & -0.337 & 0.817 & 0.6 \\
PC & 5 & 0 & -1 & 0.858 & 0.0138 & 0.532 & 0.0133 & 0.514 & 0.756 \\
\hline Classic & 2 & +1 & +1 & 0.772 & -0.00292 & 0.215 & -0.00877 & 0.645 & 1. \\
PC & 2 & +1 & +1 & 0.780 & -0.00358 & 0.212 & -0.0107 & 0.638 & 1. \\
\hline Classic & 1 & -1 & -1 & 0.703 & -0.0932 & 0.746 & -0.0901 & 0.721 & 0.733 \\
PC & 2 & -1 & -1 & 0.887 & 0.140 & 0.485 & 0.136 & 0.469 & 0.756 \\
\hline
\end{tabular}

Figure 9 shows those for principal component proxy. The correlation coefficient for the classic method was 0.977 , as shown in the scatter plot in Figure 8 a. The bias was -0.0063 parts-per-million by volume (ppmv) while the root-meansquare (RMS) error was 0.22 ppmv. In the histogram error plot in Figure $8 \mathrm{~b}$. the errors have been normalized by the original error estimates for the POAM III retrievals. This makes it easy to compare residuals with the estimated errors for the original ozone estimates. When this is done, the bias becomes -0.023 while the RMS error is 1.18. In other words, if the original error statistics are to be believed, the accuracy of ozone interpolates is almost as good as the original estimates from the POAM satellite.

The correlation coefficient for the PC proxy method was 0.987 , as shown in the scatter plot in Figure $8 \mathrm{k}$. The bias was $0.0039 \mathrm{ppmv}$ while the RMS error was 0.16 ppmv. The normalized values are 0.021 for the bias and 0.86 for RMS error. In other words, residuals for the reconstructed ozone are better, on average, than the error bounds for the original retrievals! On the other hand, the PC proxy method, while more accurate, appears to be less stable as the pair of negative values in Figure 9a suggest.

\subsection{Sonde validation}

In the second validation exercise, ozone fields were reconstructed from all available POAM data and compared with radio-sonde measurements. Figure 10 shows the results for the classic proxy tracer reconstruction validated against ozone sonde data from the WOUDC, while figure 11 shows the same for the PC proxy method. Correlation for the classic method stands at 0.76 , with a bias of $-0.074 \mathrm{ppmv}$ and a RMS error of $0.59 \mathrm{ppmv}$ while the PC proxy method provides a correlation coefficient of 0.91 , a bias of $-0.0040 \mathrm{ppmv}$ and a RMS error of 0.36 ppmv. 

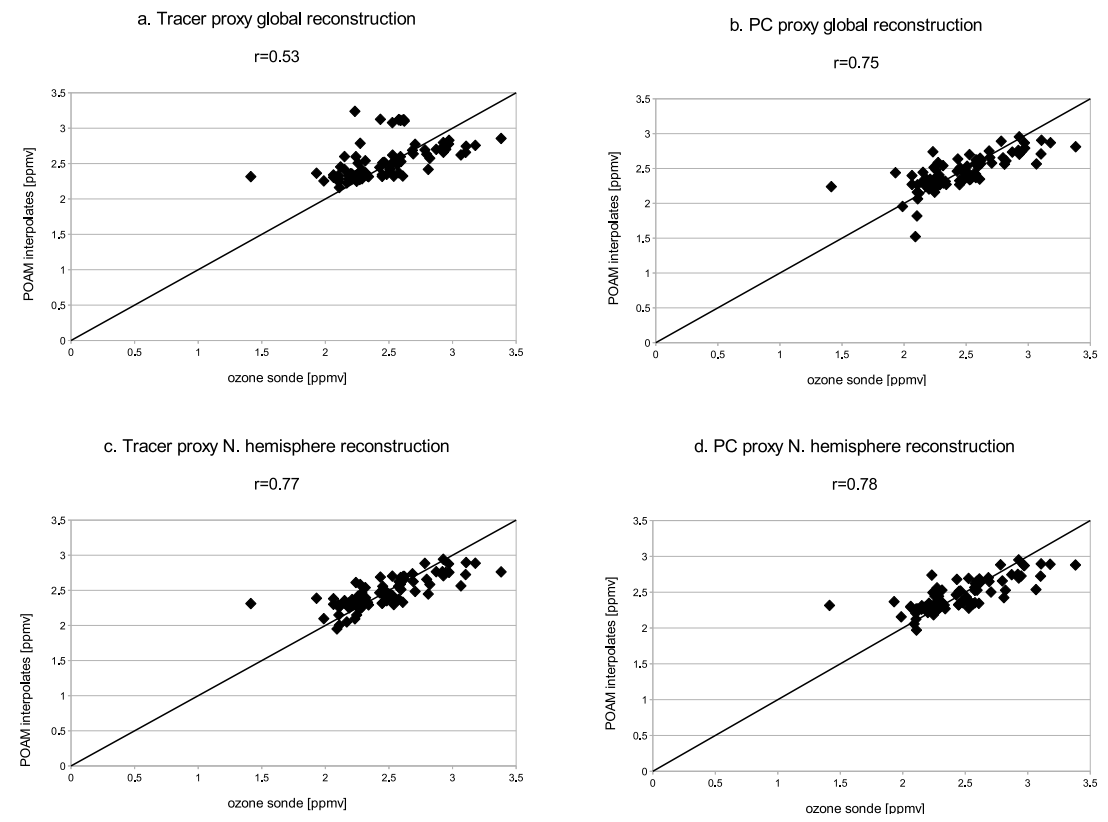

Figure 12: Validation of ozone reconstruction against ozone sonde measurements in the Northern hemisphere: a. global reconstruction classic proxy tracer; b. global reconstruction PC proxy; c. classic proxy tracer Arctic POAM III only; d. PC proxy Arctic POAM III only. 

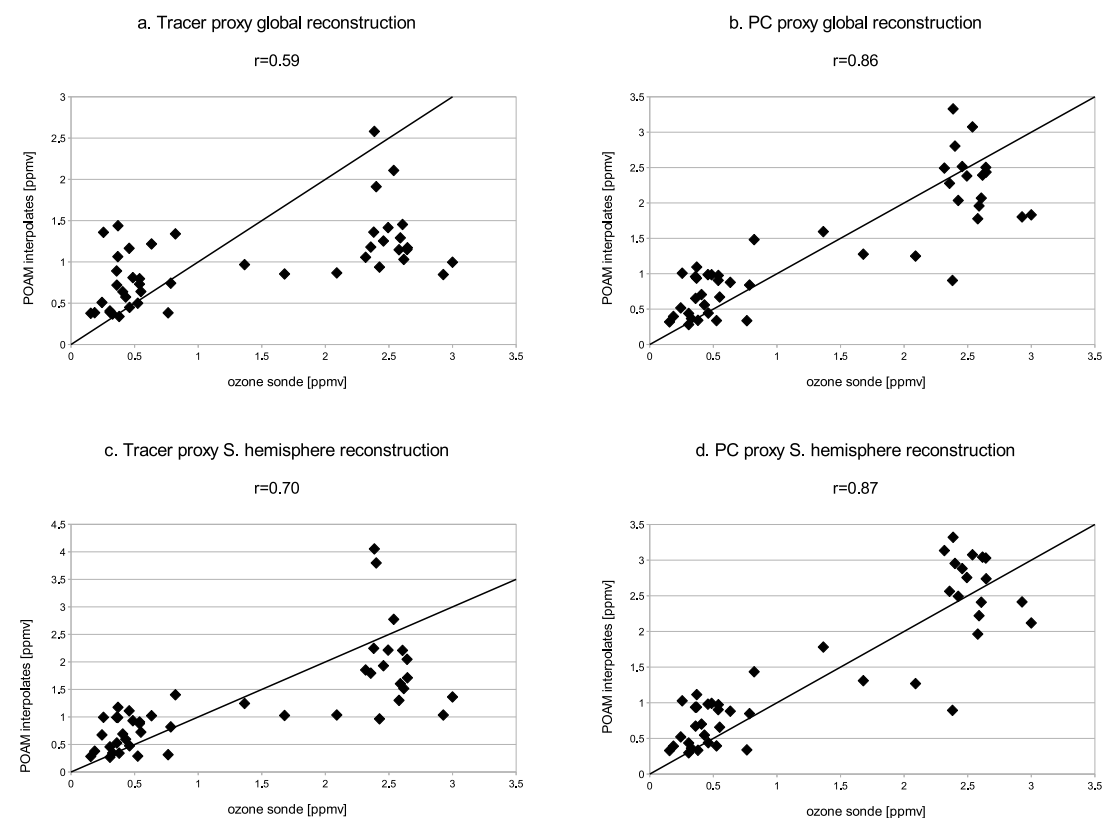

Figure 13: Validation of ozone reconstruction against ozone sonde measurements in the Southern hemisphere: a. global reconstruction classic proxy tracer; b. global reconstruction PC proxy; c. classic proxy tracer Antarctic POAM III only; d. PC proxy Antarctic POAM III only. 
For the two case studies discussed up to this point, the PC proxy method definitely has the edge. On the other hand, because ozone values tend to be higher in the Northern hemisphere than they are in the South, skill scores may be unnaturally high. As pointed out in the previous section, the original proxy tracer was typically only applied to one hemisphere at a time. For a more well-rounded comparison, we should also apply them to only the Northern or Southern hemisphere. Meanwhile, to get a better idea of the skill of the methods, we should likewise restrict comparisons to only a single hemisphere.

To get a more rigorous evaluation of each method we shall first confine them to the Northern hemisphere since ozone values there tend to fall in a narrower range of between 2 and 3 ppmv or so. To this end, Figure 12 shows scatter plots of only the Northern hemisphere sonde launches as compared to the POAM interpolates. In Figures $12 \mathrm{k}$. and $12 \mathrm{~b}$. the reconstruction was done globally but comparisons restricted to the Northern hemisphere. Skill scores for the restricted comparisons show a correlation coefficient of 0.53 , a bias of 0.078 ppmv and a RMS error of $0.30 \mathrm{ppmv}$ for the classic proxy and a correlation coefficient of 0.75 , a bias of -0.014 and a RMS error of 0.22 for the PC proxy. Figures $12 \mathrm{r}$. and $12 \mathrm{k}$. show results for interpolates for which the reconstruction was done in the Northern hemisphere using Arctic POAM III measurements only. Scores improve considerably for the classic proxy tracer and now almost match PC proxy. Skills scores for the Northern Hemisphere reconstruction show a correlation coefficient of 0.77 , a bias of -0.0029 ppmv, and a RMS error 0.22 ppmv for the classic tracer proxy and a correlation coefficient of 0.78 , a bias of -0.0035 ppmv, and a RMS error of 0.21 ppmv for the PC proxy. For the N. hemisphere reconstruction, only two (2) principal components were used in $\mathrm{PC}$ proxy.

Results for the Southern hemisphere, shown in Figure 13, are far more favourable for the PC proxy method. For global reconstruction restricted to the S. hemisphere, classic proxy returned a correlation coefficient of 0.59 , a bias of -0.35 ppmv and a RMS error of 0.85 ppmv while PC proxy gave a correlation coefficient of 0.86 , a bias of 0.014 and a RMS error of 0.53 . For S. hemisphere reconstruction, the numbers are: a correlation coefficient of 0.70 , a bias of $0.0093 \mathrm{ppmv}$ and a RMS error of $0.75 \mathrm{ppmv}$ for classic proxy while PC returned a correlation coefficient of 0.89 , a bias of 0.14 and a RMS error of 0.49 . Once again, only two principal components were used in the hemispherical PC proxy reconstruction. For the classic proxy tracer, hemispherical interpolates were to only first-, rather than second-order $(N=1)$.

All results for the sonde validation are summarized in Table 1 including relative bias, normalized RMS error and FAC2. Both relative bias and normalized RMS are normalized by the standard deviation of the measurement data. FAC2 is the fraction of estimates that are within a factor of 2 of the measurement values. 


\section{Discussion and conclusions}

The raw skill scores listed in the previous section show the PC proxy method to be more accurate than the classic proxy tracer method. Differences are largest when the reconstruction is performed globally. When the reconstruction is performed over only a single hemisphere, differences are smaller but nonetheless significant. The two methods are almost the same in the Northern hemisphere but in the Southern hemisphere PC proxy is superior even when the PC proxy reconstruction is performed globally while the classic proxy tracer is performed only in the Southern hemisphere.

We surmise that part of the advantage lies in having more degrees of freedom or at least (in the case of $k \leq N+1$ ), more meaningful degrees of freedom: more than one possible tracer configuration is represented. Moreover, because it is using information from shorter time scales, it is better able to fit more active tracers that are changing more rapidly, as ozone would be in the Southern hemisphere during winter time.

Unfortunately, because of these extra degrees of freedom, the method can also be more unstable. This is observed in negative values that showed up first in the cross-validation exercise in Section 5.1, but also in ringing and regions of negative concentration towards the equator in some of the reconstructed fields. This is to be expected: the POAM III data covers only a very small latitude band so that values derived near the equator could be considered an extrapolation and are vulnerable to "over-fitting". Such instabilities also appeared in the classic method, although less often. They don't affect the validation results so much because most of the radiosonde launch stations are in the higher latitudes, as seen in Figure 2,

In PC proxy, instabilities can be reduced by using fewer principal components which reduces the number of degrees of freedom. Unfortunately, this also tends to reduce the accuracy in the areas where the method does not fail. A good example of this phenomenon is the classic proxy in the Southern hemisphere. Here we have used only a first-order fit because while a second-order fit produced higher correlation scores, it also returned a much larger bias and RMS error. Stability is traded off for greater potential precision. The best remedy would be either to have more measurements sampling more of the globe or to simply not use interpolates far outside the range of the measurements.

The ability to accurately combine information over the whole globe would appear to be the biggest advantage of the method. As compared to the earlier method, PC proxy allows for seamless reconstruction even across the equator. Note that there is very little difference between the global reconstructions and those restricted to a single hemisphere, especially in the Southern hemisphere. Even though there isn't a lot of cross-talk between the two hemispheres, the the greater degrees of freedom in the PC proxy method overcomes this. It would be instructive to test the method on an instrument that samples more broadly and using more principal components.

Principal component proxy tracer reconstruction is shown to be a powerful technique that has many of the advantages of prognostic assimilation models 
but with less of the associated complexity. In particular, there is no need for explicitly modelled sources and sinks. It has the advantage over the classic proxy tracer method because it takes into account more than one possible configuration of a passive tracer, operates over shorter time scales and has more parameters to tune for optimal accuracy and stability. It is also more accurate when performed over the entire Earth rather than a single hemisphere, allowing for seamless reconstruction of global fields. It would be straightforward to adapt to three dimensional reconstruction as well as reconstruction that relies on column measurements or even un-inverted level 1 satellite measurements rather than point measurements. Consider the vector, $\vec{w}_{i}$, in Equation (14) to be a set of weights for integrating a column of air or performing a radiative transfer simulation rather than a set of interpolation coefficients.

\section{Acknowledgements}

Thanks to the National Center for Environmental Prediction and the National Center for Atmospheric Research for the reanalysis data used in the simulations. Thanks also to World Ozone and Ultraviolet Data Center and Environment Canada for ozone sonde data. And thanks especially to my former colleagues at the Naval Research Laboratory for POAM III ozone data.

Contour maps were created with Generic Mapping Tools (GMT) while scatter plots and historgrams were done in Open Office.

\section{References}

Allen, D. R. and Nakamura, N. (2003). Tracer Equivalent Latitude: A Diagnostic Tool for Isentropic Transport Studies. Journal of the Atmospheric Sciences, 60:287-303.

Butchart, N. and Remsberg, E. E. (1986). The area of the stratospheric polar vortex as a diagnostic for tracer transport on an isentropic surface. Journal of the Atmospheric Sciences, 43:1319-1339.

Golub, G. H. and van Loan, C. F. (1996). Matrix Computations. Johns Hopkins University Press.

Hare, E. W., Carty, E. J., and Wardle, D. I. (2000). Guide to the WMO/GAW world ozone data centre. Technical report, Meteorological Service of Canada, Environment Canada.

Hoskins, B. J., McIntyre, M. E., and Robertson, A. W. (1985). On the use and significance of isentropic potential vorticity maps. Quarterly Journal of the Royal Meteorological Society, 111:877-946.

Kalnay, E., Kanamitsu, M., Kistler, R., Collins, W., Deaven, D., Gandin, L., Iredell, M., Saha, S., White, G., Woollen, J., Zhu, Y., Chelliah, M., Ebisuzaki, 
W., Higgins, W., Janowiak, J., Mo, K., Ropelewski, C., Wang, J., Leetmaa, A., Reynolds, R., Jenne, R., and Joseph, D. (1996). The NCEP/NCAR 40year reanalysis project. Bull. Amer. Meteor. Soc., 77:437-470.

Lehoucq, R. B. and Scott, J. A. (1996). An Evaluation of Software for Computing Eigenvalues of Sparse Nonsymmetric Matrices. Technical Report MCSP547-1195, Argonne National Laboratory.

Lucke, R. L., Korwan, D. R., Bevilacqua, R. M., Hornstein, J. S., Shettle, E. P., Chen, D. T., Daehler, M., Lumpe, J. D., Fromm, M. D., Debrestian, D., Neff, B., Squire, M., Knig-Langlo, G., and J. Davies, J. (1999). The Polar Ozone and Aerosol Measurement(POAM) III instrument and early validation results. Journal of Geophysical Research, 104(D15):18785-18799.

Lumpe, J. D., Bevilacqua, R. M., Hoppel, K. W., and Randall, C. E. (2002). POAM III retrieval algorithm and error analysis. Journal of Geophysical Research, 107(D21):ACH5.1-ACH5.32.

Mills, P. (2004). Following the Vapour Trail: a Study of Chaotic Mixing of Water Vapour in the Upper Troposphere. Master's thesis, University of Bremen.

Mills, P. (2009). Isoline retrieval: An optimal method for validation of advected contours. Computers $\&$ Geosciences, 35(20):2020-2031.

Ott, E. (1993). Chaos in Dynamical Systems. Cambridge University Press.

Press, W. H., Teukolsky, S. A., Vetterling, W. T., and Flannery, B. P. (1992). Numerical Recipes in C. Cambridge University Press, 2nd edition.

Randall, C. E., Lumpe, J. D., Bevilacqua, R. M., Hoppel, K. W., Fromm, M. D., Salawitch, R. J., Swartz, W. H., Lloyd, S. A., Kyro, E., von der Gathen, P., Claude, H., Davies, J., DeBacker, H., Dier, H., Molyneux, M. J., and Sanchoi, J. (2002). Reconstruction of three-dimensional ozone fields using POAM III during SOLVE. Journal of Geophysical Research, 107(D20):8299-8312.

Rodgers, C. D. (2000). Inverse Methods for Atmospheric Sounding: Theory and Practice. World Scientific.

Tang, Y. R., Kleeman, R., and Miller, S. (2006). ENSO predictability of a Fully Coupled GCM Model Using Singular Vector Analysis. Journal of Climate, 19(14):3361-3377.

\section{A Model properties}

\section{A.1 Mass conservation}

Suppose that:

$$
\sum_{i} q_{i}=\text { const. }
$$


This will be true for non-divergent flows on equal area grids. Then:

$$
\begin{aligned}
\sum_{i} \sum_{j} r_{i j} q_{j} & =\sum_{j} q_{j} \\
\sum_{j} q_{j}\left(\sum_{i} r_{i j}-1\right) & =0
\end{aligned}
$$

Therefore:

$$
\sum_{i} r_{i j}=1
$$

If (411) is true, then:

$$
\frac{\mathrm{d}}{\mathrm{d} t} \sum_{i} q_{i}=0
$$

is also be true. Continuing:

$$
\begin{aligned}
\sum_{i} \frac{\mathrm{d} q_{i}}{\mathrm{~d} t} & =0 \\
\sum_{i} \sum_{j} a_{i j} q_{j} & =0 \\
\sum_{j} q_{j} \sum_{i} a_{i j} & =0
\end{aligned}
$$

which shows the second part of (26) and (27):

$$
\sum_{i} a_{i j}=0
$$

\section{A.2 Diffusion and the Lyapunov spectrum}

A discrete tracer mapping will always require some amount of diffusion. This means that the tracer configuration will tend towards a uniform distribution over time, that is, it will "flatten out." We can show that, given the constraint in (41), a tracer field with all the same values has the smallest magnitude. Suppose there are only two elements in the tracer vector, $\vec{q}=\{q, q\}$. The magnitude of the vector is:

$$
|\vec{q}|=\sqrt{q^{2}+q^{2}}=\sqrt{2} q
$$

Now we introduce a separation between the elements, $2 \Delta q$, that nonetheless keeps the sum of the elements constant:

$$
\begin{aligned}
|q+\Delta q, q-\Delta q| & =\sqrt{(q+\Delta q)^{2}+(q-\Delta q)^{2}} \\
& =\sqrt{2} \sqrt{q^{2}+(\Delta q)^{2}} \geq \sqrt{2} q
\end{aligned}
$$


This will generalize to higher-dimensional vectors. In general, we can say that:

$$
\vec{q} R^{T} R \vec{q} \leq|\vec{q}|^{2}
$$

Implying that for the eigenvalue problem,

$$
\begin{aligned}
R^{T} R \vec{v} & =s^{2} \vec{v} \\
s^{2} & \leq 1
\end{aligned}
$$

Therefore the Lyapunov exponents are all either zero or negative. Note however that this does not constitute a proof; the actual proof is more involved.

To prove (54) from (53), we first expand $\vec{q}$ in terms of the right singular values, $\left\{\vec{v}_{i}\right\}$ :

$$
\vec{q}=\sum_{i} c_{i} \vec{v}_{i}
$$

where $\left\{c_{i}\right\}$ are a set of coefficients. Substituting this into the left-hand-side of (53):

$$
\begin{aligned}
\vec{q} R^{T} R \vec{q} & =\sum_{i} c_{i} \vec{v}_{i} \sum_{i} c_{i} s_{i}^{2} \vec{v}_{i} \\
& =\sum_{i} \sum_{j} c_{i} c_{j} s_{i}^{2} \vec{v}_{i} \vec{v}_{j} \\
& =\sum_{i} \sum_{j} c_{i} c_{j} s_{i}^{2} \delta_{i j} \\
& =\sum_{i} c_{i}^{2} s_{i}^{2}
\end{aligned}
$$

where $\delta$ is the Kronecker delta. Similarly, we can show that:

$$
\vec{q} \cdot \vec{q}=\sum_{i} c_{i}^{2}
$$

If we assume that $s_{i} \leq 1$ for every $i$, then:

$$
\sum_{i} c_{i}^{2} s_{i}^{2} \leq \sum_{i} c_{i}^{2}
$$

since each term on the left side is less-than-or-equal-to the corresponding term on the right side. Note that in order for the inequality in (61) to be broken, at least one singular value must be greater-than one. Therefore (53) is true for every $\vec{q}$ if-and-only-if (54) is true for every $s$. In the language of set theory and first-order logic:

$$
\forall \vec{q} \in \Re^{n}\left(|R \vec{q}|^{2} \leq|\vec{q}|^{2}\right) \Longleftrightarrow \forall s \in \Re \mid R^{T} R \vec{v}=s^{2} \vec{v}(s \leq 1)
$$




\section{B Deviation from equal area}

Here we calculate the ratio between the largest and smallest grid boxes in the azimuthal equidistant coordinate system. First we show that there is no distortion at the pole:

$$
\begin{aligned}
\lim _{x \rightarrow 0, y \rightarrow 0}\left(\frac{\mathrm{d} s}{\mathrm{~d} x}\right)^{2} & =\lim _{x \rightarrow 0, y \rightarrow 0}\left(\frac{\mathrm{d} s}{\mathrm{~d} y}\right)^{2} \\
& =\lim _{x \rightarrow 0, y \rightarrow 0} \frac{1}{r^{2}}\left[\frac{R_{E}^{2}}{r^{2}} \sin ^{2}\left(\frac{r}{R_{E}}\right) y^{2}+x^{2}\right] \\
& =\frac{1}{r^{2}}\left[\frac{R_{E}^{2}}{r^{2}}\left(\frac{r}{R_{E}}\right)^{2} y^{2}+x^{2}\right] \\
& =1
\end{aligned}
$$

hence the ratio between projected and unprojected areas is 1 . Grid areas become progressively smaller the further from the pole you get. Since the projection is hemi-spherical, $r$ takes on a maximum value at the equator:

$$
r=\pi R_{E} / 2
$$

Hence the largest possible values for $x$ and $y$ are:

$$
x=y=\frac{\pi R_{E}}{2 \sqrt{2}}
$$

which represents a point on the equator along a diagonal from the origin in the projected coordinate system. The metric coefficients can be calculated:

$$
\begin{aligned}
\left(\frac{\mathrm{d} s}{\mathrm{~d} x}\right)^{2} & =\left(\frac{\mathrm{d} s}{\mathrm{~d} y}\right)^{2} \\
& =\frac{4}{\pi^{2} R_{e}^{2}}\left[\frac{4 R_{E}^{2}}{\pi^{2} R_{E}^{2}} \sin ^{2}\left(\frac{\pi R_{E}}{2 R_{E}}\right) \frac{\pi^{2} R_{E}^{2}}{8}+\frac{\pi^{2} R_{E}^{2}}{8}\right] \\
& =\frac{1}{\pi^{2} R_{E}^{2}}\left(2 R_{E}^{2}+\frac{\pi^{2} R_{E}^{2}}{2}\right) \\
& =\frac{2}{\pi^{2}}+\frac{1}{2} \\
& \approx 0.703
\end{aligned}
$$

